\title{
In vivo cardiac diffusion tensor imaging in free-breathing conditions
}

\author{
Hongjiang Wei ${ }^{1^{*}}$, Magalie Viallon ${ }^{1,2}$, Benedicte M Delattre ${ }^{1}$, Vinay M Pai ${ }^{3}$, Han Wen $^{3}$, Hui Xue ${ }^{4}$, \\ Christoph Guetter ${ }^{4}$, Marie-Pierre Jolly ${ }^{4}$, Pierre Croisille ${ }^{1,5}$, Yuemin Zhu ${ }^{1}$ \\ From 16th Annual SCMR Scientific Sessions \\ San Francisco, CA, USA. 31 January - 3 February 2013
}

\section{Background}

The fiber structure of the human heart contributes significantly to efficient ventricular function in the presence of disease. DTI provides a non-invasive approach for the three-dimensional depiction of the myocardial fiber architecture. The biggest problem for in vivo cardiac DTI is the signal loss caused by motion. Recently, to cope with human physiological motion problem, a robust method called PCATMIP was proposed (Rapacchi, Invest radiol 2011) that uses principal component analysis (PCA) filtering to improve the signal-to-noise ratio (SNR) and temporal maximum intensity projection (TMIP) approach to compensate the signal loss. While performing cardiac DTI during subject's breath-hold may be not realistic to apply in clinical routine, achieving acquisitions during subject free-breathing represents an ultimate objective. In this study, our objective was to obtain in vivo DTI parameters of the human heart with free-breathing.

\section{Methods}

To cope with intensity fluctuations arising due to motion, our strategy was to acquire multiple diffusion weighted (DW) images at different time points during the diastole in each consecutive cardiac cycle; after each time frame was acquired, the trigger delay was increased by $10 \mathrm{~ms}$. At each trigger delay, we obtained 12 direction DW images and $b=0$. We acquired 10 DT-MRI slices across the whole heart. The total scan time is about 20 minutes at an average heart rate of $60 \mathrm{bpm}$. The MRI parameters are: $\mathrm{TE} / \mathrm{TR}=51 / 100 \mathrm{~ms}$, spatial resolution $=2.6 \times 2.6 \times 6 \mathrm{~mm} 3$, acceleration rate $=2$ (GRAPPA), partial Fourier $=6 / 8$, base resolution matrix $=90 \times 160$, and $\mathrm{b}=200 \mathrm{~s} / \mathrm{mm} 2$. Free-breathing DWI scans were then registered using a non-rigid registration algorithm that preserves high accuracy and consistency of the data. Finally, PCATMIP algorithm was applied to the registered images to obtain motion-reduced ones.

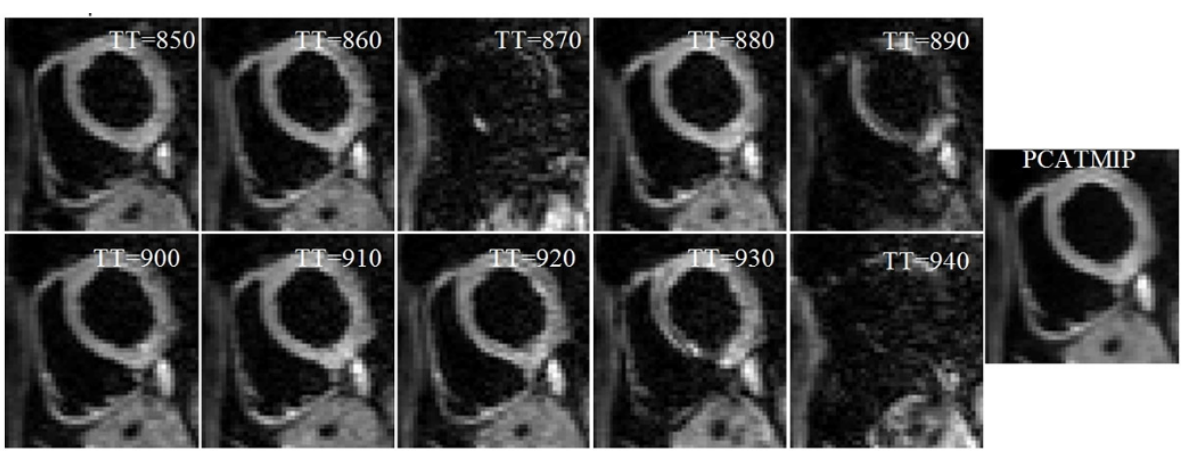

Figure 1 Free-breathing cardiac DW images from 10 repetitions acquired at different time points and processed using PCATMIP.

${ }^{1}$ Creatis; CNRS (UMR 5220), Inserm (U1044); Insa Lyon, Villeurbanne, France

Full list of author information is available at the end of the article

(c) 2013 Wei et al; licensee BioMed Central Ltd. This is an Open Access article distributed under the terms of the Creative Commons Attribution License (http://creativecommons.org/licenses/by/2.0), which permits unrestricted use, distribution, and reproduction in any medium, provided the original work is properly cited. 


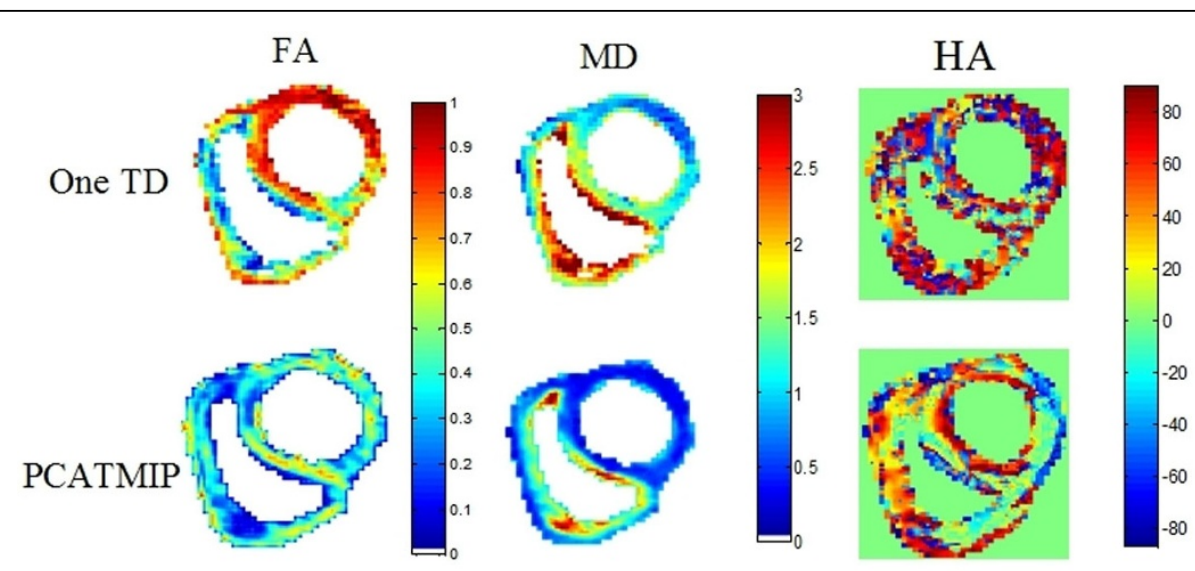

Figure $2 \mathrm{FA}, \mathrm{MD}$, and HA maps comparison between one trigger delay and PCATMIP, the chosen reference trigger delay was the one that exhibited the highest homogeneity and signal intensity in the myocardium.

\section{Results}

Lost signal was recovered and the intensity of the DW images was substantially enhanced (Fig. 1). After processing by PCATMIP, both FA $(0.43 \pm 0.05)$ and MD $(0.75$ $\pm 0.12 \times 10-3 \mathrm{~mm} 2 / \mathrm{s})$ are smaller than those obtained from 1 TD acquisition $(0.56 \pm 0.05$ and $1.37 \pm 0.16 \times 10-3$ $\mathrm{mm} 2 / \mathrm{s}$, respectively) measured over the LV. Coherent helix angle (HA) variations across the ventricle's wall were observed (Fig. 2). The myocardial fiber orientation of the LV showed a circularly symmetric pattern, which reflects the rotation characteristic of cardiac fibers.

\section{Conclusions}

This study demonstrates the feasibility of in vivo cardiac DTI in healthy volunteers. The PCATMIP can be used to minimize the motion-induced signal loss. The proposed acquisition and processing scheme allow to obtain the in vivo DTI parameters while the subject was freely breathing, which opens interesting perspectives for in vivo cardiac DTI clinical applications.

\section{Funding}

This work was supported by the French ANR 2009 (under ANR-09-BLAN-0372-01).

\footnotetext{
Author details

${ }^{1}$ Creatis; CNRS (UMR 5220), Inserm (U1044)i, Insa Lyon, Villeurbanne, France. 'Department of Radiology, University Hospitals of Geneva, Geneva, Switzerland. ${ }^{3}$ Imaging Physics Lab, BBC/NHLBI/NIH, Bethesda, MD, USA. ${ }^{4}$ Siemens Corporate Research, Princeton, NJ, USA. 5 Jean-Monnet University, Saint-Etienne, France.
}

Published: 30 January 2013
doi:10.1186/1532-429X-15-S1-P231

Cite this article as: Wei et al:: In vivo cardiac diffusion tensor imaging in free-breathing conditions. Journal of Cardiovascular Magnetic Resonance 2013 15(Suppl 1):P231.
Submit your next manuscript to BioMed Central and take full advantage of:

- Convenient online submission

- Thorough peer review

- No space constraints or color figure charges

- Immediate publication on acceptance

- Inclusion in PubMed, CAS, Scopus and Google Scholar

- Research which is freely available for redistribution

Submit your manuscript at www.biomedcentral.com/submit
C Biomed Central 